\title{
Ultrahigh Frequency Data Liquidity Duration Estimation: A Case Study of Chinese A Shares
}

\author{
Jianhui Yuan, ${ }^{1,2}$ Yu Pan, ${ }^{1}$ and Xin Zhang $^{3}$ \\ ${ }^{1}$ Department of Finance and Insurance, Nanjing University of Information Science and Technology, Nanjing 210044, China \\ ${ }^{2}$ Department of Geography and Program in Planning, University of Toronto, Toronto, ON, Canada M5S $3 G 3$ \\ ${ }^{3}$ School of Economics and Management, Nanjing University of Science and Technology, Nanjing 210094, China
}

Correspondence should be addressed to Jianhui Yuan; jianh.yuan@utoronto.ca

Received 13 July 2014; Accepted 16 October 2014

Academic Editor: Ufuk Yolcu

Copyright (C) 2015 Jianhui Yuan et al. This is an open access article distributed under the Creative Commons Attribution License, which permits unrestricted use, distribution, and reproduction in any medium, provided the original work is properly cited.

\begin{abstract}
Liquidity has always been a hot spot for researchers of financial market microstructures. Analysis of liquidity is of great significance for investors and market regulators. Ultrahigh frequency data records the whole dynamic change of the trading process, so it has advantages in depicting the market microstructure. This study analyzes Asian emerging market equities liquidity using ultrahigh frequency data. We used various forms of WACD models and let trading duration be indicators of liquidity. Through the residual test, we were able to select the best model to describe the overall liquidity.
\end{abstract}

\section{Introduction}

High frequency data are collected based on a precise daily timescale. Tick-by-tick data (the stock transaction data of each tick) and tick data (the stock transaction data of each 3$5 \mathrm{sec}$.) are two kinds of typical financial high frequency data. The tick-by-tick data, which contains more information on transaction events, is called ultrahigh frequency data, and this is the data used in this research. Arithmetic transactions (also called programmed transactions) of financial high frequency data were pioneered by the practical exploration work of several famous Wall Street investment banks in the 1980s, and the practical effect of their work, particularly their record high profits, was highly praised by participants in the financial markets. The exploration of these techniques is still in the trial stage in Asian emerging market. In China, it began to emerge after high frequency data were formally disclosed by the Shanghai Stock Exchange in 2006. However, the theoretical estimation, analysis, and research in respect of ultrahigh frequency data have been slow to develop.

The liquidity of the stock market is a key factor in the activities of investors. Amihud and Mendelson [1] stated: "Liquidity is the all of the market." They thought that, for investors who will make a choice between cash and securities, the value of liquidity will be assessed for any number of reasons and investors are more willing to pay for liquidity. Liquidity is always a hot spot for those conducting researches in the financial market microstructure. Ultrahigh frequency data record the information in respect of each tick of transactions undertaken; that is, the microdynamic change of the whole market is recorded, so the liquidity constructed on ultrahigh data will be the dominating index.

The purpose of our research is to estimate the liquidity of ultrahigh frequency data for Chinese A shares by the transaction volume duration (time for the market to absorb a certain volume of transactions). A delayed multiphase WACD model was used to fit and analyze the liquidity index and to describe the trends and characteristics of liquidity changes in Chinese A shares for a day.

\section{Literature Review}

The irregularity of time intervals is a key characteristic of ultrahigh frequency data. The estimation and analysis of the liquidity of ultrahigh frequency data in and out of China have been mainly performed on the description of a time interval and are generally based on the ACD model 
(Autoregressive Conditional Duration Model). The initial work done by Engle and Russell [2] was praised as a great contribution to financial theory. In an ACD model, the time intervals between each two transaction events are estimated and analyzed as random variables, and the transaction event can be described by transaction volume, transaction value, and bid-asked spread. These transaction events are the key points for theoretical research on the centralized estimation variable of investor's transaction activities and the financial market microstructure. Engle and Lange [3] introduced a price duration based method in which the change of the asked price was used as a transaction event to estimate the rate of transactions absorbed by the market, and the time varying liquidity of the US market was analyzed using an ACD model to prove the applicability of this model. Engle [4] forecasted and explained the changing mode of daily stock market liquidity using ACD and GARCH models with ultrahigh frequency data. Bauwens and Giot [5] introduced the use of Log-ACD models to estimate stock liquidity and verified their estimations by the price durations of three stocks listed on the New York Stock Exchange.

Presently, scholars in China only use daily stock density and cycle-spanned data for their liquidity research. In China, research on the liquidity of (ultra) high frequency data began in 2000. The late entry into research in this area was due to the difficulty in data collection.

ACD models have been used by Chinese scholars in the estimation of high frequency data liquidity from 2002. Their research on the liquidity of high frequency data has been mainly realized by two methods: one is to study the influence of microprice fluctuations on liquidity and the other is to study the interaction between transaction volume changes and liquidity.

Foreign research has mostly been carried out using the first method above. Research in China is also based on foreign experience, that is, by estimating the timing change of liquidity by price fluctuation. Jin and Yang [6] analyzed the influencing factors of liquidity in the Shanghai stock market and found that the stock liquidity index can be distinctly explained by profit fluctuations and stock prices. Chen et al. [7] used a dynamic ACD model to study the price duration of high frequency data of the Shanghai and Shenzhen stock markets and found that price durations are inversely proportional to the fluctuations in Chinese stock markets.

Further research has shown that the quotation mechanism in China is different to the foreign mechanism. The foreign mechanism is a market maker rule, whereas, in China, it is a call auction rule. The change of stock price per second is usually 0.01 Yuan, so it is difficult to compare the fluidity of two high liquidity stocks using the price fluctuations.

$\mathrm{Bu}$ et al. [8] initially used the fluctuations of transaction volumes to describe the liquidity of high frequency data. They introduced the liquidity index of transaction volume duration and found that this index has a high demonstrative effectiveness. Cao et al. [9] compared the descriptions of five ACD models to the liquidity index transaction volume duration sequence and found that the $\operatorname{WACD}(2,1)$ had the best fitness of the five models. Wang [10] used WACD, EACD, and Log-ACD models to fit and estimate the transaction volume duration sequence. They found that the WACD model had the best fitness to the transaction time interval sequence of high frequency data and that the Log-ACD model was more suitable for fitting and estimating the long term developing trend of duration.

To sum up, foreign research on the liquidity of high frequency data started earlier than in China but it has mostly been centered on the influence of price fluctuations. Research on the liquidity of high frequency data in China started only recently and there is a large room for improvement in the future. Meanwhile, data processing and model selection using the transaction volume duration as the index for estimation are still in the exploration stage. Research by models is basically limited to the various extensions of the $\operatorname{ACD}(1,1)$ model. This study explores the analysis and demonstrative research of multiphase delayed extended ACD models for the fitness of transaction duration.

\section{Concept and Estimation of Liquidity}

3.1. Concept and Traditional Estimation Index of Liquidity. Liquidity is a key characteristic of the market microstructure and ultrahigh frequency data contain the true market information at a maximum level; that is, the data contain the trades processed that are usually regarded as noise in traditional low frequency data research. Our study used ultrahigh frequency data to develop a demonstrative study on market microstructure.

In the security market, if an order that is submitted is found to be hard to execute or requires a long time frame to be executed, we will naturally think that the market liquidity is insufficient. As O'Hara [11] said: "Liquidity is easy to identify but hard to define." There is no globally uniform definition for liquidity. Schwartz [12] defined liquidity as the capacity for finishing the transaction rapidly at a reasonable price. Glen [13] asserted that liquidity was the capacity for rapid transactions without any sharp price changes. Amihud [14] defined liquidity as the time needed to search for a suitable price.

The above definitions show that liquidity is involved with three factors: speed (transaction time), price (transaction cost), and transaction volume (large transactions can be completed rapidly at a reasonable price). Accordingly, our study on methods for estimating liquidity will be started with these three factors. According to the summary for liquidity index, the method for estimating liquidity can be divided into a price method, a transaction volume method, a time method, and a price-volume method. Each method has several estimating indexes, and the typical liquidity estimating indexes are shown in Table 1.

Current estimating indexes have all evolved and extended from typical indexes shown in Table 1. Liquidity has several factors, so the characteristics can hardly be reflected by a single index, and each method reflects only some of the liquidity characteristics in a different view. We can say that there is no uniform method to estimate liquidity and different liquidity indexes are used by scholars for different research purposes. 
TABLE 1: Typical liquidity estimating indexes.

\begin{tabular}{lll}
\hline $\begin{array}{l}\text { Estimating } \\
\text { method }\end{array}$ & Estimating indexes \\
\hline Pricing & $\begin{array}{l}\text { Spread } \\
\text { Pricing effective spread } \\
\text { Relative effective spread }\end{array}$ \\
\hline Amounting & $\begin{array}{l}\text { Market depth } \\
\text { Amounting transaction depth } \\
\text { Turnover }\end{array}$ & \\
\hline \multirow{3}{*}{ Timing } & $\begin{array}{l}\text { Execution time } \\
\text { Transaction frequency }\end{array}$ \\
& Elasticity & \\
\hline $\begin{array}{l}\text { Amount-price } \\
\text { compounding }\end{array}$ & Price impact model & $\begin{array}{l}\text { Kyle model } \\
\text { LL model }\end{array}$ \\
& Liquid ratio & $\begin{array}{l}\text { Amivest liquidity ratio } \\
\text { Martin index }\end{array}$ \\
\hline
\end{tabular}

3.2. Ultrahigh Frequency Data Liquidity Estimating Index. As in tick-by-tick transaction data, there are dozens or hundreds of different transactions per second in different time intervals. Traditional liquidity indexes are not applicable for ultrahigh frequency data. Current relevant analyses are performed with common liquidity indexes, including bid-asked spread, market depth, and duration. The duration contains more transaction information in the financial microfield. For our study, we chose the duration method, in combination with time and transaction volume, and used transaction volume duration as the index for liquidity estimation.

Duration is the time length from the beginning to the end of an event. The time interval (duration) between two transaction events can reflect the transactors' determination on transaction information and the characteristics of their transacting activity, and it is significant for explaining liquidity. The statistical characteristics and dynamic change of duration show the transaction activity and daily liquidity characteristics in the stock market.

In the transaction volume duration, the fixed transaction volume accumulated value is used as a transaction event. It denotes the time consumed in absorbing a fixed stock transaction volume in the market. The fixed transaction volume is called as a "threshold," which can be freely chosen as per the stock transaction conditions.

This study envisages that $x_{i}$ is the $i$ th transaction volume duration, and its value is the time interval between the $i$ th transaction event and the $(i-1)$ th transaction event; that is, $x_{i}=t_{i}-t_{i-1}$. We envisage that the transaction event is that the stock transaction volume is accumulated to a fixed value, that is, to the threshold, and a transaction volume duration is formed when the transaction volume from the last moment to the current moment has been accumulated to the threshold.

The transaction volume duration contains two dimensions-transaction time and transaction volume. Obviously, the length of the transaction volume duration is inversely proportional to the rate of stock liquidity. Lower transaction volume duration means better liquidity and vice versa. This research mainly analyzes the liquidity of $\mathrm{A}$ shares in China by the estimation and analysis of the whole transaction volume duration. An actual transaction has clear buying and selling directions, but the whole transaction volume duration has no clear buying and selling directions as it consists of the order absorption and activity of the whole market.

\section{General Description of ACD Model}

4.1. Principle of ACD Model. The irregularity of time intervals is in contrast with the envisaged model for fixed time interval data in current econometrics (e.g., GARCH and random dynamic modeling). The expression of fluctuation characteristics is not applicable any more. In relation to this characteristic, Engle, who introduced the ARCH model, and his partner Russell [2] further introduced the ACD model. They constructed an estimating model by using the duration as a random variable $x$. Almost all of the duration problems of current financial microtransaction events can be described by ACD models.

In the earliest ACD model, the time interval of the transaction event is transformed into a dynamic point process before it is modeled and analyzed. A standard ACD model is usually created in demonstrative research using the following method. We assume that $x_{i}$ is the $i$ th transaction volume duration and its value is the time interval between the $i$ th transaction event and the $(i-1)$ th transaction event; that is, $x_{i}=t_{i}-t_{i-1}$. Different types of duration can be created by different transaction events, such as the transaction happened, a fixed price fluctuation, and the transaction volume accumulated to a fixed value, and thus the transaction duration, price duration, and transaction volume duration can be obtained accordingly. This study uses transaction volume duration.

This is the standard ACD $(p, q)$ mode. The conditional expectation of delayed phase $q$ and the past practice of delayed phase $p$ will influence the conditional expectation of the $i$ th duration together:

$$
\begin{gathered}
E\left(x_{i} \mid x_{i-1}, x_{i-2}, \ldots, x_{1}\right)=\psi_{i}\left(x_{i-1}, x_{i-2}, \ldots, x_{1} ; \theta\right) \equiv \psi_{i}, \\
\psi_{i}=\omega+\sum_{j=1}^{p} \alpha_{j} x_{i-j}+\sum_{j=1}^{q} \beta_{j} \psi_{i-j} .
\end{gathered}
$$

4.2. Extended ACD Models. The ACD model can be developed into different ACD models according to different residual density functions. The four most common density functions are standard index distribution, Weibull distribution, Gamma distribution, and extreme value distribution. The four linear ACD models corresponding to the four residual density functions are the EACD, WACD, GACD, and BACD models, respectively.

We found that the $\operatorname{ACD}(p, q)$ model is similar to the GARCH $(p, q)$ model in the mode, and thus the ACD model can be extended with conditional duration. These are mainly the ACD-GARCH model, introduced by Ghysels and Jassik [15], the Log-ACD model, introduced by Bauwens and Giot [5], and the Threshold ACD model, introduced by Zhang et al. [16]. 
In recent years, when Chinese scholars used an ACD model to study the market microstructure, EACD, WACD, GACD, and Log-ACD models were mostly used. Demonstrative research shows that the WACD model has the best fitness to the liquidity of the Chinese stock market. The LogACD model can remove negative duration and has a favorable effect on the long term fitness estimation of duration.

This research mainly discusses the liquidity estimation of short term transaction volume duration, so the WACD model was chosen as the estimating model because it has better short term fluctuation fitness, and it mainly tries to compare the fitness of the WACD model for different delayed phases. The following is an introduction to the WACD model.

A WACD model is an ACD model when the imagined $\varepsilon_{i}$ complies with a standard Weibull distribution. The standard Weibull $(1, \gamma)$ distribution is

$$
f(x)= \begin{cases}\gamma\left(\Gamma\left(1+\frac{1}{\gamma}\right) x\right)^{\gamma-1} e^{-(\Gamma(1+1 / \gamma) x)^{\gamma},}, & \text { if } x \geq 0, \\ 0 & \text { if } x<0\end{cases}
$$

where $\gamma$ is the shape parameter of the Weibull distribution. When $\gamma=1$, it is the index distribution; when $\gamma<1$, the function of the Weibull distribution is exclusively decreasing, (i.e., the possibility of long duration is regressive); when $\gamma>1$, the function of the Weibull distribution is exclusively increasing (i.e., the possibility of long duration is increasing $\gamma)$. The possibility for long or short duration can be forecasted and described by the coefficient.

Obtained by maximum likelihood estimation, the Log likelihood function of the WACD model is

$$
\begin{gathered}
\ln L=\sum_{i=1}^{N}\left[\ln \left(\frac{\gamma}{L_{i}}\right)+\gamma \ln \left(\frac{\Gamma(1+1 / \gamma) L_{i}}{\psi_{i}}\right)\right. \\
\left.-\left(\frac{\Gamma(1+1 / \gamma) L_{i}}{\psi_{i}}\right)^{\gamma}\right] .
\end{gathered}
$$

\section{Demonstrative Research on the Liquidity of Chinese A Shares on the Shanghai Stock Exchange}

5.1. Sample Selection and Preliminary Data Process. In China, an executive meeting of the State Council released the "National Five Rules" for real estate regulation on February 20, 2013. These required each district to prepare and publish the annual price control target for newly built commercial residential houses and to strictly execute their purchase control measures in order to maintain stable house prices. After these rules were released, real estate shares were shaken intensively and investors started to pay more attention to the liquidity of real estate shares. Therefore, this event was chosen as the background for our study, and real estate shares in the Chinese A shares of the Shanghai Stock Exchange were chosen as our study objects to facilitate comparative analysis.

Sample data for this research came from the Wind database. Because the volume of tick-by-tick ultrahigh transaction data is very large (each stock has approximately
TABLE 2: Baoli Real Estate tick-by-tick ultrahigh frequency sample data.

\begin{tabular}{lccc}
\hline Time & Price & Buy/sell & Volume \\
\hline 9:30:00 & 12.29 & $\mathrm{~B}$ & 1000 \\
9:30:00 & 12.29 & $\mathrm{~B}$ & 1000 \\
9:30:00 & 12.29 & $\mathrm{~B}$ & 154 \\
9:30:00 & 12.29 & $\mathrm{~S}$ & 800 \\
9:30:00 & 12.29 & $\mathrm{~S}$ & 46 \\
9:30:00 & 12.29 & $\mathrm{~S}$ & 154 \\
9:30:00 & 12.30 & $\mathrm{~B}$ & 100 \\
9:30:02 & 12.29 & $\mathrm{~S}$ & 46 \\
9:30:02 & 12.29 & $\mathrm{~S}$ & 1000 \\
9:30:02 & 12.29 & $\mathrm{~S}$ & 354 \\
9:30:02 & 12.29 & $\mathrm{~S}$ & 2000 \\
9:30:02 & 12.29 & $\mathrm{~S}$ & 1000 \\
9:30:02 & 12.29 & $\mathrm{~S}$ & 4846 \\
9:30:02 & 12.29 & $\mathrm{~S}$ & 1000 \\
\hline
\end{tabular}

20,000 to 30,000 pieces of transaction information daily), it is difficult to process all of this data. A review of the prices of real estate company shares in the Chinese A shares of the Shanghai Stock Exchange from February to March of 2013 showed that three real estate shares were always in the top ten, namely, Baoli Real Estate (SH600048), Chengcheng Group (SH600247), and Jindi Group (SH600383).

When selecting the sample period, scholars basically use the tick data from two weeks to two months. The amount of tick-by-tick data is much more than the amount of tick data in the same time period. In order that we could predict the short term liquidity of the real estate shares more accurately, we selected the transaction data within two weeks after the "National Five Rules" are released. This most frequent trading period was from February 21, 2013 to March 6, 2013, containing 10 transaction days and 659,282 transaction records. The data contained the transaction time, transaction value, initiative transaction direction, and transaction volume (unit: share) for the transaction days. The sample size is large enough to finish the fitness and the prediction of the model that we used.

Matlab and Eviews 6.0 were the tools used for our estimations. Table 2 displays the data sample for Baoli Real Estate.

Before commencing our demonstrative analysis, the data were preliminarily processed to remove unnecessary and wrong data.

Firstly, the influence of call auctions and lunch breaks on the stock market transaction information and invalid transactions before 9:30 am, after 3:00 pm, and between 11:30 am and 1:00 pm were removed.

Secondly, transaction data relating to stock stop plate, harden, and drop stopping days were removed as they cause irregular transaction volume durations. Stock stop plate is temporarily to stop trading when a listed company's stock price rises or falls dramatically and abnormally. Harden or drop stopping is the stock market's trading system in the securities per day price to be appropriate restrictions on 
TABLE 3: Shares' volume descriptive statistics.

\begin{tabular}{lcccccc}
\hline Share & Sample number & Average volume & Largest volume & Smallest volume & Standard deviation & Dispersion rate \\
\hline SH600048 & 268,341 & $12,742.38$ & $6,715,600$ & 1 & $60,106.01$ & 4.717 \\
SH600383 & 260,533 & $14,818.45$ & $6,077,500$ & 1 & $61,808.38$ \\
SH600247 & 124,563 & $8,447.463$ & $1,128,700$ & 1 & $29,537.40$ & 3.171 \\
\hline
\end{tabular}

price movements in order to prevent the skyrocketing prices or prices plummeted, inhibiting excessive speculation. On Chinese A shares, the ups or downs limiter of the stock price each trading day is $10 \%$. When the stock price goes up to upper limit or drops to floor level, the trader cannot buy or sell stock at that price.

Lastly, obviously wrong data, such as those with negative transaction volumes or negative prices, were removed.

After the data were preliminarily processed via the above steps, 653,437 sample transaction records were obtained.

5.2. Construction of Transaction Volume Duration. The most important step in constructing the transaction volume duration is to select the threshold, that is, the fixed value of accumulated transaction volume. Different shares have different proportions of circulating and noncirculating shares, and if the same fixed threshold is selected, the duration for a stock transaction volume of less circulating shares will be prolonged, the liquidity will be poor, and the comparison will generate a large error. Therefore, scholars studying the transaction volume duration mostly use the multiple of the mean volume per transaction as the threshold to overcome the above problem and protect the comparability of individual stock liquidity.

Tick-by-tick ultrahigh frequency data record each tick of stock transaction information, and there will be several or hundreds of ticks of transaction records per second. Accordingly, we need to calculate the average trading volume of each transaction. Define MVPT as mean volume per transaction, $V_{i}$ as the trading volume of the $i$ th transaction, and $T$ as the whole amount of the transaction in the sample. The equation is as follows:

$$
\operatorname{MVPT}=\frac{\sum_{i=1}^{T} V_{i}}{T} .
$$

The threshold multiple can be set to satisfy the varying demands for research. Bu et al. [8] set the threshold to about 10 times the mean volume per transaction and Zheng [17] set the threshold to 20 times the mean volume per transaction, but they used tick high frequency data rather than tickby-tick ultrahigh frequency data. Ultrahigh frequency data fluctuates more frequently, so the method for estimation varies accordingly. If estimates are used of 10 or 20 times the mean volume per transactions, as selected by the scholars referred to above, a lot of zero transaction volume durations will be generated by the ultrahigh transaction data and it will be hard to see the changes of ultrahigh data transaction volume duration $x$.

Our study referred to the above thresholds and related references and combined these with the characteristics of
TABle 4: Sample shares' threshold.

\begin{tabular}{lcc}
\hline Share & Average volume & Threshold \\
\hline SH600048 & $12,742.38$ & $1,274,200$ \\
SH600247 & $8,447.463$ & 844,700 \\
SH600383 & $14,818.45$ & $1,481,800$ \\
\hline
\end{tabular}

ultrahigh frequency data to finally obtain a threshold multiple of 100 times the mean volume per transaction. This multiple is suitable to the ultrahigh frequency data after adequate adjustment and this threshold can distinctly describe the transaction volume duration sequence of ultrahigh frequency data.

To obtain the threshold, the transaction volumes of the three shares (unit: share) are statistically described in Table 3.

The sample statistical description shows that the sample numbers of first two shares have no distinct difference, but the sample numbers of third share are distinctly less than the first two. This means that the first two shares have a higher transaction frequency and the transaction volumes show the corresponding characteristics. Each share has a large difference between the minimum and maximum transaction volumes, which means that the distribution of transaction volume is quite unstable. The excessive dispersion rate (rate of standard difference to mean value) is much larger than 1 , which means that transaction volume happens irregularly and the fluctuation is quite large.

In this research, the threshold of the whole transaction volume duration was uniformly set to 100 times the mean volume per transaction, and the sample threshold of the three shares in Table 4 can be calculated from the mean volume per transaction in Table 3.

After the threshold was set and when the transaction volume was accumulated to the threshold for the first time, the time interval between the tick and the first tick was used as the first transaction volume duration and then used as the beginning of the next accumulation. A transaction volume duration was generated when the transaction volume accumulated to the threshold each time, and thus the transaction volume duration sequence for each share was obtained.

\subsection{Statistical Description of Transaction Volume Duration.}

The transaction volume duration sequences of the three shares were obtained by the above steps and the statistical description for the three shares in the whole transaction volume period is described in Figures 1, 2, and 3.

The analysis diagram shows that the mean volume per transaction duration values of Baoli Real Estate (SH600048), Chengcheng Group (SH600247), and Jindi Group (SH600383) in the whole transaction volume period 


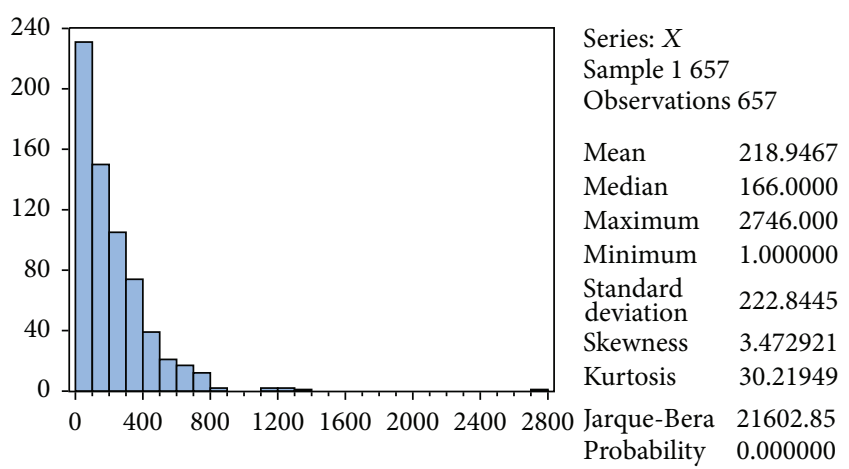

Figure 1: Baoli Real Estate (SH600048) whole transaction volume period descriptive statistics.

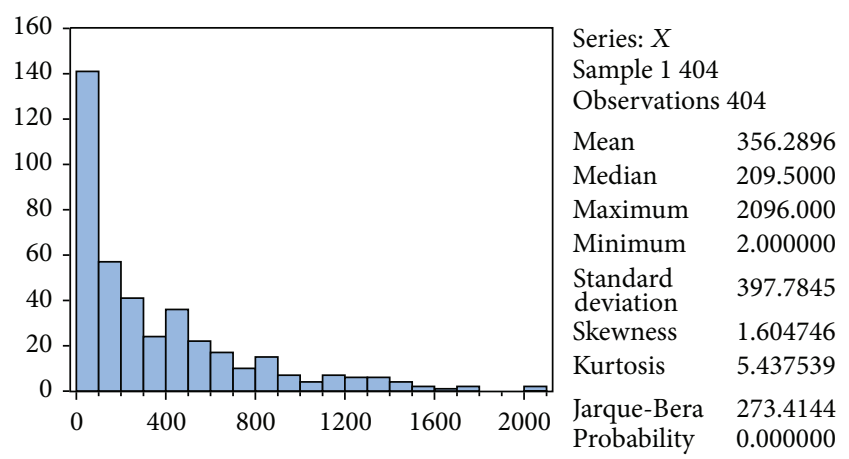

FIGURE 2: Chengcheng Group (SH600247) whole transaction volume period descriptive statistics.

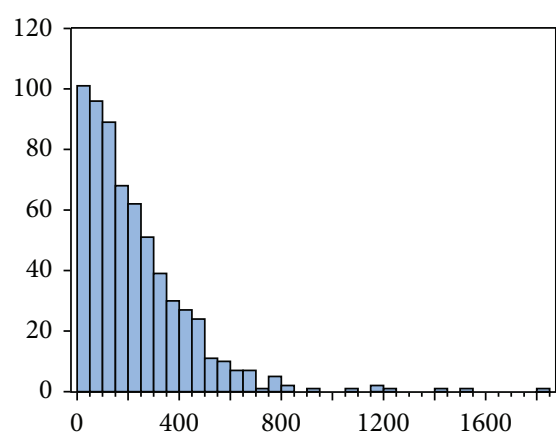

Series: $X$

Sample 1638

Observations 638

Mean $\quad 225.5361$

Median $\quad 172.5000$

Maximum $\quad 1803.000$

Minimum 1.000000

Standard $\quad 208.3586$

deviation 208.3586

Skewness 2.402617

Kurtosis 13.28627

Jarque-Bera 3426.526

Probability $\quad 0.000000$

FIGURE 3: Jindi Group (SH600383) whole transaction volume period descriptive statistics.

was $219 \mathrm{sec}$., $356 \mathrm{sec}$., and $226 \mathrm{sec}$, respectively; that is, the transaction volume reached 100 times the preset mean volume per transaction after about $219 \mathrm{sec}$., $356 \mathrm{sec}$., and $226 \mathrm{sec}$. This comparison shows that the market activity and order finishing rate of Chengcheng Group were the weakest of the three real estate shares; that is, Chengcheng Group's liquidity was weaker than that of the other two shares and Baoli Real Estate and Jindi Group had a similar liquidity level.

The standard deviation of the three shares varied from 200 to 400, which means the fluctuation of transaction

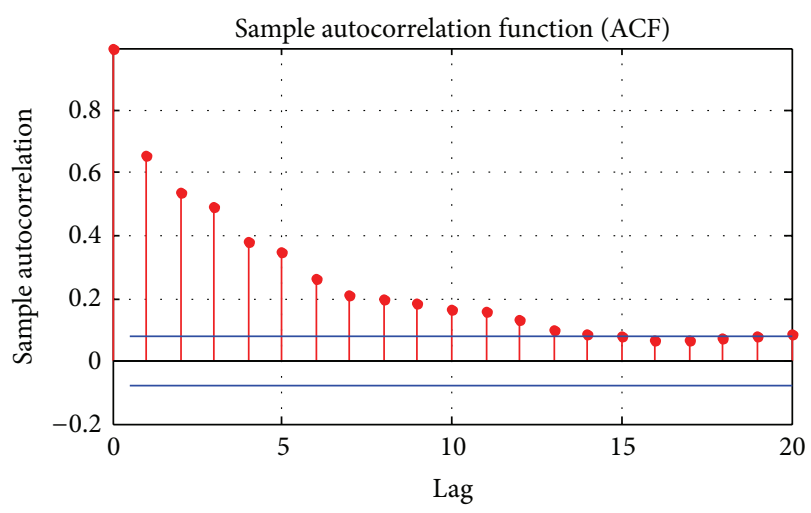

FIgURE 4: Transaction volume duration ACF of Baoli Real Estate (SH600048).

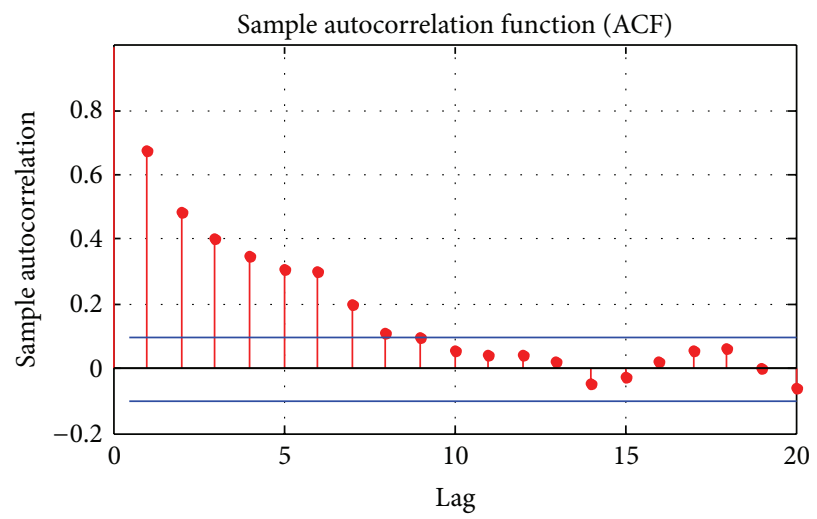

FIGURE 5: Transaction volume duration ACF of Chengcheng Group (SH600247).

volume duration was large. Statistically, the kurtosis value was much larger than 3 , which means that the value did not comply with a normal distribution. Generally, the samples had a similar statistical characteristic; that is, they had a large fluctuation and did not comply with normal distribution.

5.4. Autocorrelation of Transaction Volume Duration. Noh et al. [18] found a similar autocorrelation of market fluctuations in transaction duration data; that is, a long transaction interval is followed by a long transaction interval and a short one is followed by a short one. Figures 4, 5, and 6 use demonstrative data to determine whether the transaction volume duration had a similar autocorrelation. Figures 4, 5, and 6 show the ACF (autocorrelation factor) of the three shares in the last 20 phases delayed, and the blue line is twice the standard deviation.

The ACFs of Chengcheng Group (SH600247) and Jindi Group (SH600383) were similar to the ACF of Baoli Real Estate (SH600048). The ACFs were quite large in a phase before they dropped sharply in the following phases. They stabilized at about 10 phases delayed and maintained beyond twice the standard deviation after 20 phases delayed. Therefore, we can see that the three shares showed a strong 


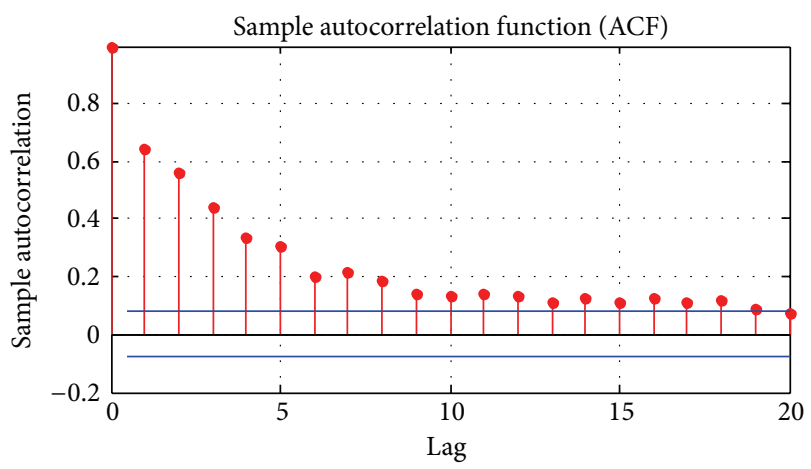

FIgURE 6: Transaction volume duration ACF of Jindi Group (SH600383).

sequence autocorrelation during the whole transaction volume duration. The sequence autocorrelation means that a long transaction duration will be followed by a long duration and a short one will be followed by a short one.

The degree of freedom of the Ljung-Box statistics was 39.9968 at the significance level of $0.5 \%$. Table 5 shows that the Ljung-Box statistics of the three shares in the whole transaction volume duration were all larger than the critical value. The original expectation that there would be no autocorrelation of the transaction volume duration sequence was obviously not met, which provides clear support for our decision to study the transaction volume duration in China using an ACD model.

5.5. Model Estimating and Fitting. In this research, a multiphase delayed WACD model was used for the modeling of transaction volume duration sequences. The verification of transaction volume duration sequence autocorrelation shown in Table 5 shows that the partial correlation factors of the three shares exceeding twice the standard deviation were at a delayed phase of 1 to 2 , so $\operatorname{WACD}(1,1)$, WACD $(1,2)$, $\operatorname{WACD}(2,1)$, and $\operatorname{WACD}(2,2)$ were used to fit the duration and a delayed model with the best fitness being obtained via comparison.

The estimation results for the parameters of the above four ACD models are shown in Tables 6, 7, 8, and 9. These results show that each sequence value of $\alpha+\beta$ is close to 1. According to the research by Engle and Russell [2], we thought that indicated a result that was highly durative during the whole transaction volume duration, and it can be effectively explained by the models. The highly durative transaction volume duration shows a long duration followed by a long duration and a short one followed by a short one; that is, the transaction volume duration process has concentrations. Because of this characteristic in transaction volume duration, we conclude that there is an intense clustering of liquidity in the Chinese stock market; that is, a high liquidity followed by a high liquidity and a low liquidity followed by a low liquidity.

Additionally, according to the Weibull function, when the expectation on the Weibull distribution is applied and the estimated value of coefficient $\gamma$ is smaller or equal to 1 , it means that the duration risk is decreased or stabilized; when
TABLE 5: Ljung-Box statistic of transaction volume duration ACF.

\begin{tabular}{lccc}
\hline Share & SH600048 & SH600247 & SH600383 \\
\hline LB $(20)$ & 989.75 & 509.66 & 917.75 \\
\hline
\end{tabular}

TABLE 6: Whole transaction volume duration WACD $(1,1)$ estimate.

\begin{tabular}{lcccc}
\hline & $\omega$ & $\alpha$ & $\beta$ & $\gamma$ \\
\hline SH600048 & 5.8223 & 0.5301 & 0.4665 & 1.6891 \\
SH600247 & 11.5155 & 1.0097 & 0.0986 & 1.2789 \\
SH600383 & 9.4413 & 0.6472 & 0.3399 & 1.7824 \\
\hline
\end{tabular}

TABLE 7: Whole transaction volume duration $\operatorname{WACD}(1,2)$ estimate.

\begin{tabular}{ccccc}
\hline & $\omega$ & $\alpha$ & $\beta$ & $\gamma$ \\
\hline SH600048 & 4.5587 & 0.5105 & {$[0.5505,-0.0549]$} & 1.6921 \\
SH600247 & 10.016 & 0.9659 & {$[-0.0319,0.1387]$} & 1.3322 \\
SH600383 & 8.6559 & 0.6542 & {$[0.3118,0.0259]$} & 1.7825 \\
\hline
\end{tabular}

TABLE 8: Whole transaction volume duration $\operatorname{WACD}(2,1)$ estimate.

\begin{tabular}{lcccc}
\hline & $\omega$ & $\alpha$ & $\beta$ & $\gamma$ \\
\hline SH600048 & 5.0016 & {$[0.5129,0.0492]$} & 0.4442 & 1.6927 \\
SH600247 & 5.9176 & {$[0.9609,-0.3748]$} & 0.4652 & 1.3054 \\
SH600383 & 8.0842 & {$[0.6532,-0.0422]$} & 0.3814 & 1.7824 \\
\hline
\end{tabular}

TABLE 9: Whole transaction volume duration $\operatorname{WACD}(2,2)$ estimate.

\begin{tabular}{ccccc}
\hline & $\omega$ & $\alpha$ & $\beta$ & $\gamma$ \\
\hline SH600048 & -0.1088 & {$[0.6177,0.3176]$} & {$[-0.1996,0.3610]$} & 1.6506 \\
SH600247 & 8.5713 & {$[0.9820,0.1456]$} & {$[-0.1770,0.1488]$} & 1.3008 \\
SH600383 & 0.098 & {$[0.6654,-0.6576]$} & {$[1.3395,-0.3472]$} & 1.7699 \\
\hline
\end{tabular}

the coefficient $\gamma$ is distinctly larger than 1 , it means that the duration risk is increased. The coefficient $\gamma$, after modeling for all durations in this research, was larger than 1 . This means that the transaction volume duration risk is increased, the possibility of long duration is enlarged, the stock liquidity becomes lower, and the influence of current liquidity to future liquidity is decreased in the Weibull mode.

\section{Verification}

To verify that these four models effectively describe the dynamic change characteristics of transaction volume duration, we used the research of Engle and Russell [2], who determined that the fitness of a model to duration can be verified by ACD with the standardized residual deviation. The standardized residual deviation of a WACD model is

$$
\widehat{\varepsilon}_{i}=\frac{x_{i}}{\psi_{i}}
$$

where $\widehat{\psi}_{i}$ is the maximum likelihood estimated value.

The residual deviation is an unpredictable part in the model. If the sequence of residual deviation is verified to be a white noise sequence, it means that this model can describe the autoregressive structure of the duration successfully; that is, it means that the model has a favorable fitting effect and 
TABLE 10: Transaction volume duration WACD $(1,1)$ residual ACF.

\begin{tabular}{lcccc}
\hline Lag & AC & PAC & Q-Stat & Probability \\
\hline 1 & 0.031 & 0.031 & 0.624 & 0.429 \\
2 & -0.086 & -0.087 & 5.476 & 0.065 \\
3 & 0.022 & 0.027 & 5.785 & 0.123 \\
4 & -0.102 & -0.112 & 12.693 & 0.013 \\
5 & 0.006 & 0.019 & 12.717 & 0.026 \\
6 & -0.081 & -0.105 & 17.125 & 0.009 \\
7 & -0.114 & -0.101 & 25.750 & 0.001 \\
8 & -0.047 & -0.073 & 27.208 & 0.001 \\
9 & -0.015 & -0.028 & 27.359 & 0.001 \\
10 & -0.011 & -0.040 & 27.439 & 0.002 \\
11 & 0.019 & -0.005 & 27.676 & 0.004 \\
12 & 0.005 & -0.022 & 27.692 & 0.006 \\
13 & -0.036 & -0.061 & 28.547 & 0.008 \\
14 & -0.033 & -0.065 & 29.284 & 0.010 \\
15 & -0.009 & -0.036 & 29.339 & 0.015 \\
\hline
\end{tabular}

TABLE 11: Transaction volume duration $\operatorname{WACD}(1,2)$ residual LjungBox testing.

\begin{tabular}{lcccc}
\hline Lag & AC & PAC & Q-Stat & Probability \\
\hline 1 & 0.059 & 0.059 & 2.264 & 0.132 \\
2 & -0.095 & -0.099 & 8.217 & 0.016 \\
3 & 0.008 & 0.020 & 8.259 & 0.041 \\
4 & -0.102 & -0.114 & 15.113 & 0.004 \\
5 & 0.001 & 0.019 & 15.115 & 0.010 \\
6 & -0.083 & -0.109 & 19.648 & 0.003 \\
7 & -0.116 & -0.100 & 28.613 & 0.000 \\
8 & -0.049 & -0.072 & 30.199 & 0.000 \\
9 & -0.014 & -0.029 & 30.335 & 0.000 \\
10 & -0.008 & -0.040 & 30.381 & 0.001 \\
11 & 0.021 & -0.005 & 30.670 & 0.001 \\
12 & 0.006 & -0.023 & 30.693 & 0.002 \\
13 & -0.036 & -0.061 & 31.541 & 0.003 \\
14 & -0.033 & -0.063 & 32.294 & 0.004 \\
15 & -0.010 & -0.035 & 32.359 & 0.006 \\
\hline
\end{tabular}

forecasting performance. The Ljung-Box statistic is a key tool for verifying whether the residual deviation sequence is a white noise sequence. The original expectation is that all the autocorrelation coefficients of the sequence are zero, and the selective expectation is that at least one of the autocorrelation coefficients is insignificantly zero. The original expectation will be refused if the Ljung-Box $Q$ statistic is larger than the critical value, or if it is accepted, the sequence is white noise, and the model is successfully fitted. The following table used the whole transaction volume duration of Baoli Real Estate (SH500048) as an example to verify that the standard residual deviations of the four models are white noise, as shown in Tables 10, 11, 12, and 13 .

When the significance level of the Ljung-Box $Q$ statistics is $1 \%$, the critical value at a freedom degree of 15 is 30.578 . Table 10 shows that the $Q$ statistics of the whole transaction
TABLE 12: Transaction volume duration $\operatorname{WACD}(2,1)$ residual LjungBox testing.

\begin{tabular}{lcccc}
\hline Lag & AC & PAC & Q-Stat & Probability \\
\hline 1 & 0.055 & 0.055 & 2.014 & 0.156 \\
2 & -0.093 & -0.097 & 7.776 & 0.020 \\
3 & 0.012 & 0.023 & 7.872 & 0.049 \\
4 & -0.101 & -0.113 & 14.592 & 0.006 \\
5 & 0.002 & 0.019 & 14.595 & 0.012 \\
6 & -0.082 & -0.109 & 19.120 & 0.004 \\
7 & -0.116 & -0.100 & 28.101 & 0.000 \\
8 & -0.049 & -0.072 & 29.687 & 0.000 \\
9 & -0.015 & -0.028 & 29.835 & 0.000 \\
10 & -0.009 & -0.040 & 29.892 & 0.001 \\
11 & 0.020 & -0.005 & 30.160 & 0.001 \\
12 & 0.005 & -0.023 & 30.179 & 0.003 \\
13 & -0.036 & -0.061 & 31.043 & 0.003 \\
14 & -0.034 & -0.064 & 31.801 & 0.004 \\
15 & -0.010 & -0.035 & 31.867 & 0.007 \\
\hline
\end{tabular}

TABLE 13: Transaction volume duration $\operatorname{WACD}(2,2)$ residual LjungBox testing.

\begin{tabular}{lcccc}
\hline Lag & AC & PAC & Q-Stat & Probability \\
\hline 1 & 0.028 & 0.028 & 0.535 & 0.465 \\
2 & -0.019 & -0.020 & 0.784 & 0.676 \\
3 & 0.019 & 0.020 & 1.016 & 0.797 \\
4 & -0.078 & -0.080 & 5.069 & 0.280 \\
5 & 0.004 & 0.009 & 5.079 & 0.406 \\
6 & -0.074 & -0.079 & 8.733 & 0.189 \\
7 & -0.108 & -0.101 & 16.453 & 0.021 \\
8 & -0.044 & -0.050 & 17.750 & 0.023 \\
9 & -0.015 & -0.015 & 17.905 & 0.036 \\
10 & -0.010 & -0.021 & 17.968 & 0.056 \\
11 & 0.018 & 0.004 & 18.189 & 0.077 \\
12 & 0.004 & -0.009 & 18.202 & 0.110 \\
13 & -0.032 & -0.050 & 18.902 & 0.126 \\
14 & -0.032 & -0.052 & 19.578 & 0.144 \\
15 & -0.009 & -0.022 & 19.639 & 0.186 \\
\hline
\end{tabular}

value duration of Baoli Real Estate were all lower than the critical value by the fitting of WACD $(1,1)$, the residual deviation is white noise, and this model can fit the transaction volume duration. Tables 11,12 , and 13 are the residual deviation verification result when the transaction volume duration sequence is fitted by a WACD model with changed delayed phase.

The residual deviation verifications for $\operatorname{WACD}(1,2)$ and WACD $(2,1)$ are similar; both have Ljung-Box $Q$ statistics larger than LB (15) (i.e., 30.578) in 2 or 3 delayed phases, so the fitting effect is not favorable. Table 13 shows that the residual deviation of WACD $(2,2)$ is white noise and the fitting effect is favorable.

In view of the residual deviation autocorrelation verification for whole transaction volume duration, most of 


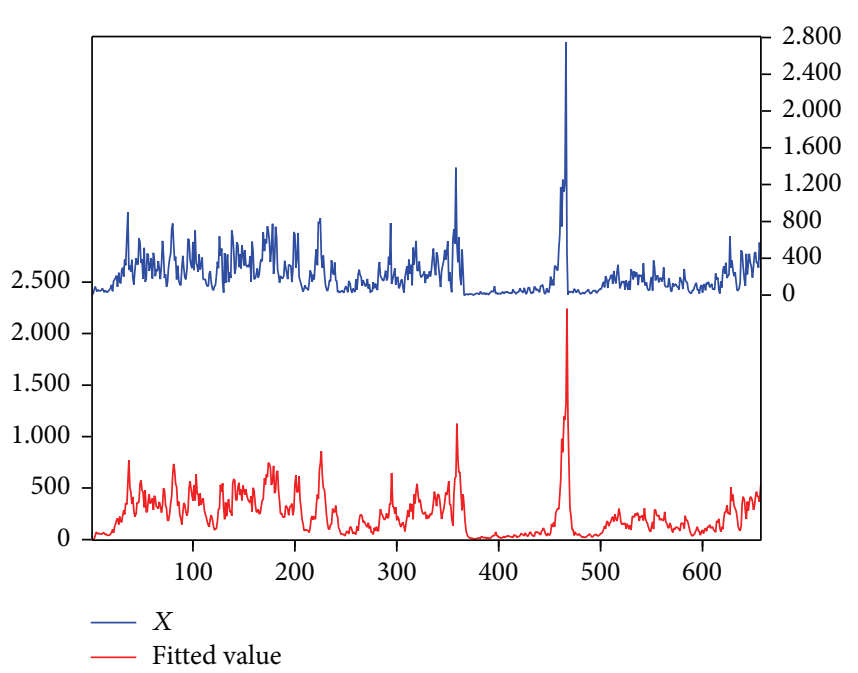

Figure 7: Outcome of WACD $(2,2)$ fitting of Baoli Real Estate (SH600048).

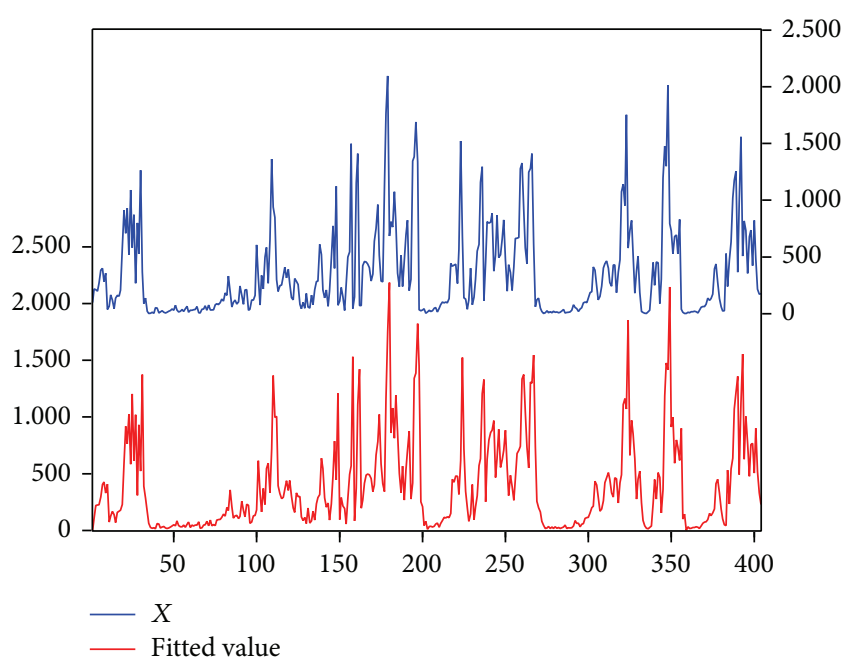

Figure 8: Outcome of WACD $(2,2)$ fitting of Chengcheng Group (SH600247).

the Ljung-Box statistics for the residual deviation of this stock are within the critical value, the fitting effect of the WACD $(2,2)$ model is better than that of the WACD $(2,1)$ model, and WACD $(2,1)$ is more effective than WACD $(1,2)$. The autocorrelation figures for the whole transaction volume duration of the other two stocks are similar to Baoli Real Estate, and thus they are not described here in detail.

The shares selected for this research are all large A shares on the Shanghai Stock Exchange. The sample is large, so some Ljung-Box statistics beyond the critical value are reasonable. For the whole liquidity, $\operatorname{WACD}(2,2)$ shows a better forecasting performance.

The relative fitness and forecasting performance of WACD $(2,2)$ are verified by the fitting figures (Figures 7,8 , and 9$)$ of the three shares in the $\operatorname{WACD}(2,2)$ model $(x$ at the top is the actual value and the fitted value is at the bottom).

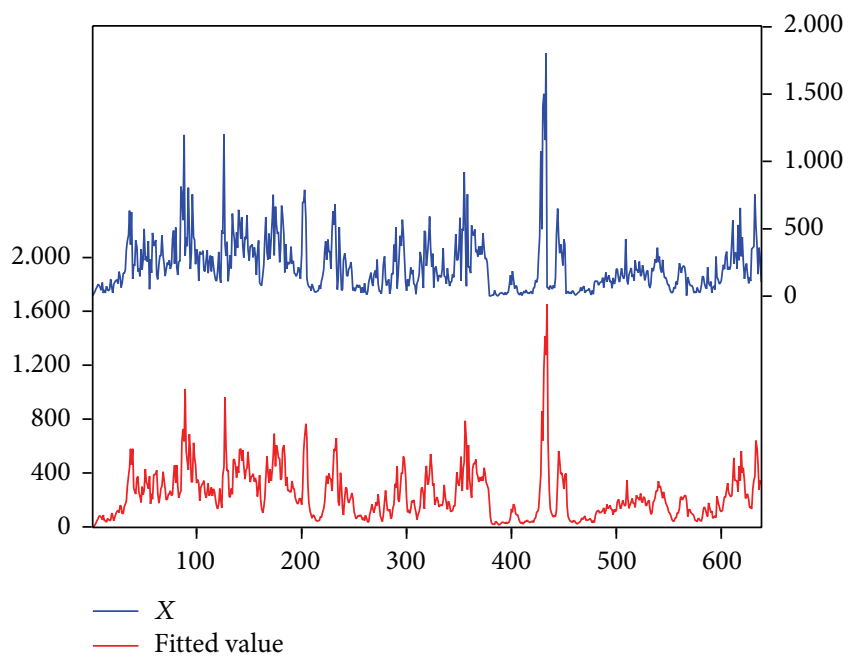

Figure 9: Outcome of WACD $(2,2)$ fitting of Jindi Group (SH600383).

Figures 7,8 , and 9 show that the $\operatorname{WACD}(2,2)$ model has an ideal fitting effect to the whole transaction volume durations of the three shares. It effectively forecasts the short period fluctuation trend but is not so effective in forecasting the long term trend.

Fitting Figures 7, 8, and 9 show that the transaction volume duration trends of Baoli Real Estate and Jindi Group are centralized at a stable level, and the trend of Chengcheng Group fluctuates a lot, but there is also some irregularity. The transaction volume durations of Baoli Real Estate are mostly below $400 \mathrm{sec}$., which is the time selected by most people to conclude their transaction, and a wrong judgment on stock transaction completion can be avoided by judging this time interval. The duration is long in some intervals, which means that the stock liquidity is poor in this period, and it is hard to exchange the stock in this duration. The transaction volume durations of Jindi Group are mostly $500 \mathrm{sec}$., which is generally similar to Baoli Real Estate. Chengcheng Group shows a distinct trend; the fitting effect is not favorable compared with that of the other two shares. The fitting figure shows a typical liquidity clustering effect; that is, a high liquidity followed by a high liquidity and a low liquidity followed by a low liquidity.

By the estimating and fitting of our WACD models to the stock transaction volume duration, we can see the liquidity change of a share over the course of a day; we can see when transactions are active and when they are down and thus we can find a valuable investing point. Therefore, estimating the stock ultrahigh frequency data liquidity is useful for us to understand the stock market microstructure, and we can ascertain the instant stock liquidity in a brand new angle of view other than by any traditional low frequency data, such as the daily closing price. Research on stock ultrahigh frequency data liquidity enables us to more clearly understand the true operation of the stock market. This research is an important starting point for studying the financial market microstructure. 


\section{Conclusion}

This research analyzes the liquidity of Chinese stock market as a representative of Asian emerging market. It estimated the liquidity in view of the time and transaction volume of the transaction volume duration based on existing research in and out of China. Our main findings were as follows.

First, we found that the threshold selection is very important for model fitness, and the threshold value will be fixed by the data characteristics. Multiphase delayed WACD $(1,1)$, WACD $(1,2)$, WACD $(2,1)$, and WACD $(2,2)$ models were used for the fitness comparison of duration, and the fitting effect of the WACD $(2,2)$ model was found to be the best.

Second, in the model fitting process, we found that the duration characteristics of ultrahigh frequency data can reflect the transaction clustering. According to this characteristic in transaction volume duration, we determined that there is an intense clustering of liquidity in the Chinese stock market; that is, a high liquidity followed by a high liquidity and a low liquidity followed by a low liquidity.

Third, the coefficient $\gamma$ after modeling for all durations in our study was larger than 1 . This means that the transaction volume duration risk is increased, the possibility of long duration is enlarged, the stock liquidity becomes lower, and the influence of current liquidity on future liquidity is decreased in the Weibull mode.

For the three real estate shares selected, the model fitness for Chengcheng Group was poor because of the threshold selection, and the circulating stock capital levels of Baoli Real Estate and Jindi Group were larger than that of Chengcheng Group. This threshold is a multiple of mean volume per transaction, but the difference in individual shares still exists, so a better threshold selecting method will be a direction for future research.

Our study was handicapped by the difficulty experienced in ultrahigh frequency data processing and had some other shortcomings. First, the sample for this research was small; the results satisfied our requirements with some economic meaning, but the results would have been more stringent if the sample capacity could have been further enlarged. Second, a simple linear model may not be effective enough for the research on ACD models. Extending future research into complicated nonlinear models, which would be challenging, may produce better results.

\section{Conflict of Interests}

The authors declare that there is no conflict of interests regarding the publication of this paper.

\section{References}

[1] Y. Amihud and H. Mendelson, "Liquidity and asset prices: financial management implications," Financial Management, vol. 17, no. 1, pp. 5-15, 1988.

[2] R. F. Engle and J. R. Russell, "Autoregressive conditional duration: a new model for irregularly spaced transaction data," Econometrica, vol. 66, no. 5, pp. 1127-1162, 1998.
[3] R. F. Engle and J. Lange, "Measuring, forecasting and explaining time varying liquidity in the stock market," Tech. Rep., National Bureau of Economic Research, 1997.

[4] R. F. Engle, "The econometrics of ultra-high-frequency data," Econometrica, vol. 68, no. 1, pp. 1-22, 2000.

[5] L. Bauwens and P. Giot, "The logarithmic ACD model: an application to the bid-ask quote process of three NYSE stocks," Annuls of Economy and Statistic, vol. 60, pp. 117-149, 2000.

[6] Y. Jin and W. Yang, "Empirical of liquidity affected factor on Shanghai stock market," Journal of Financial Research, vol. 6, no. 264, pp. 12-21, 2002.

[7] M. Chen, G. Wang, G. Wu, and X. Jiang, "The application of ACD-GARCH model in Chinese stock market," Chinese Statistical Research, no. 11, pp. 60-62, 2003.

[8] F. Bu, W. Zhang, and W. He, "Research on stock liquidity based on ultrahigh frequency data," Statistics \& Decision, vol. 2, pp. 24-26, 2005.

[9] Y. Cao, W. Qiu, and S. Liu, "Research on intraday liquidity in security market of China based on ACD model," Chinese Journal of Management Science, vol. 14, no. SI, pp. 351-355, 2006.

[10] Z. Wang, The Research of Chinese Stock Market Liquidity Measure Based on High-frequency Data, Tianjin University, 2009.

[11] M. O'Hara, Market Microstructure Theory, Blackwell Publishers, Cambridge, UK, 1995.

[12] R. A. Schwartz, Equity Markets: Structure, Trading and Performance, Harper and Row, New York, NY, USA, 1998.

[13] J. Glen, "A Introduction to the Microstructure of Emerging Markets," Discussion Paper, International Finance Corporation, Washington, DC, USA, 1994.

[14] Y. Amihud, "Illiquidity and stock returns: cross-section and time-series effects," Journal of Financial Markets, vol. 5, no. 1, pp. 31-56, 2002.

[15] E. Ghysels and J. Jassik, "GARCH for irregurally spaced financial data: the ACD-GARCH model," Studies in Nonlinear Dynamics \& Econometrics, vol. 2, no. 4, pp. 1-19, 1998.

[16] M. Zhang, J. Russell, and R. Tsay, "A nonlinear conditional autoregressive duration model with applications to financial transactions data," Journal of Econometrics, vol. 104, no. 1, pp. 179-207, 2001.

[17] L. Zheng, Research on Decision-Making Quantifying of Algorithmic Trading in Chinese Stock Market Based on High-Frequency Data, University of Science and Technology of China, 2010.

[18] J. Noh, R. F. Engle, and A. Kane, "Forecasting volatility and option prices of the S\&P 500 index," The Journal of Derivatives, vol. 2, no. 1, pp. 17-30, 1994. 


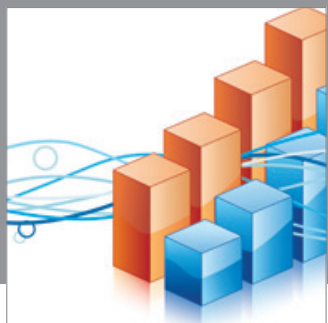

Advances in

Operations Research

mansans

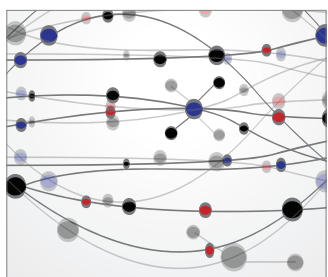

The Scientific World Journal
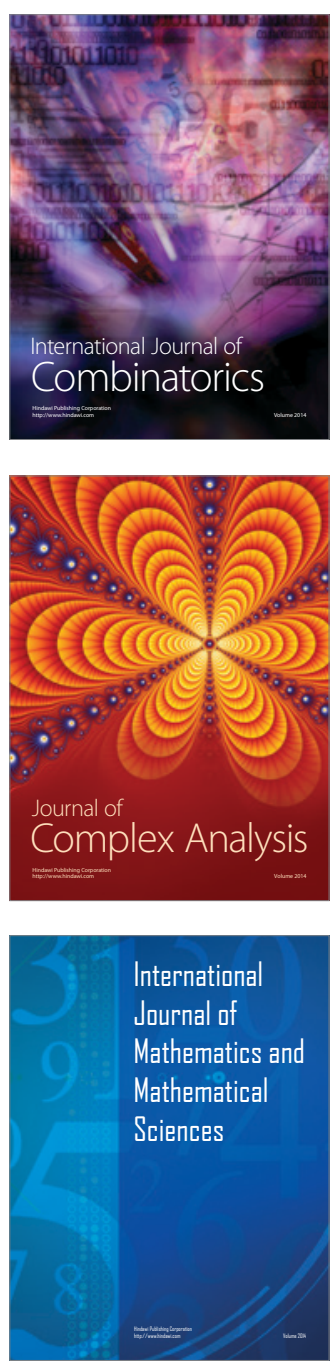
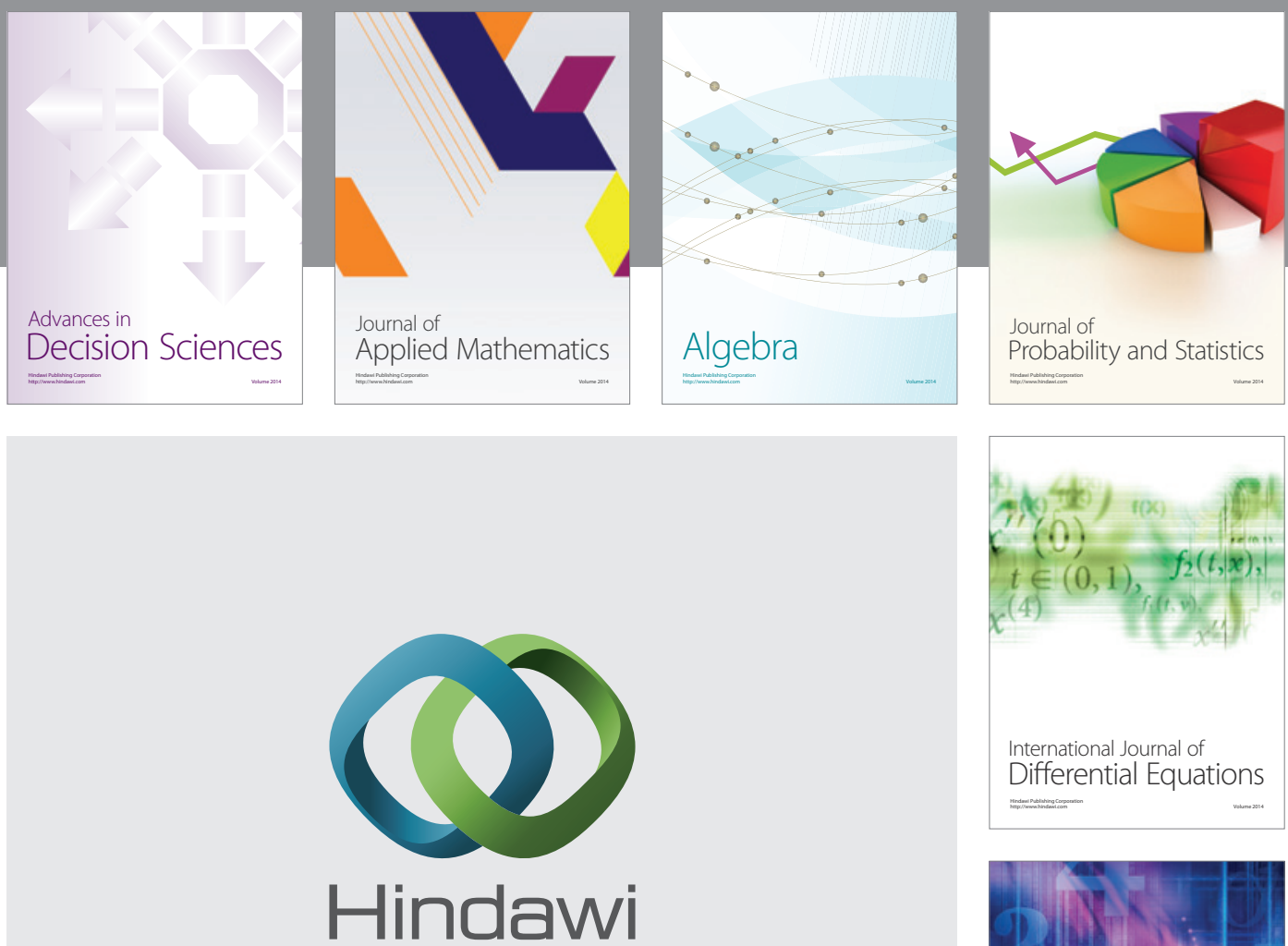

Submit your manuscripts at http://www.hindawi.com
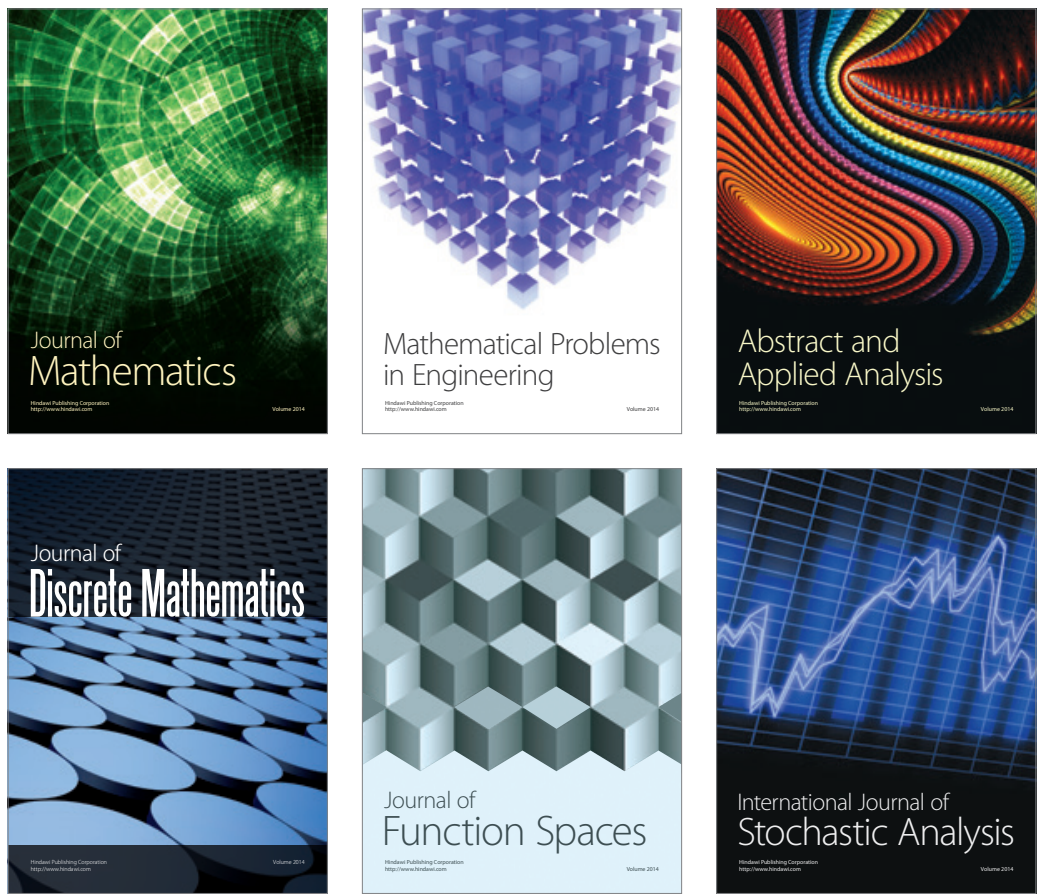

Journal of

Function Spaces

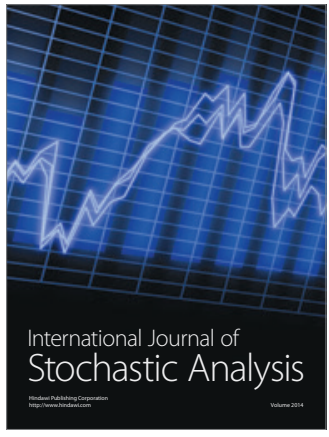

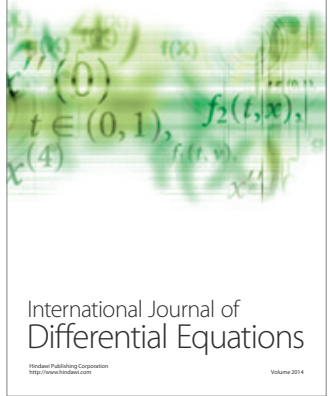
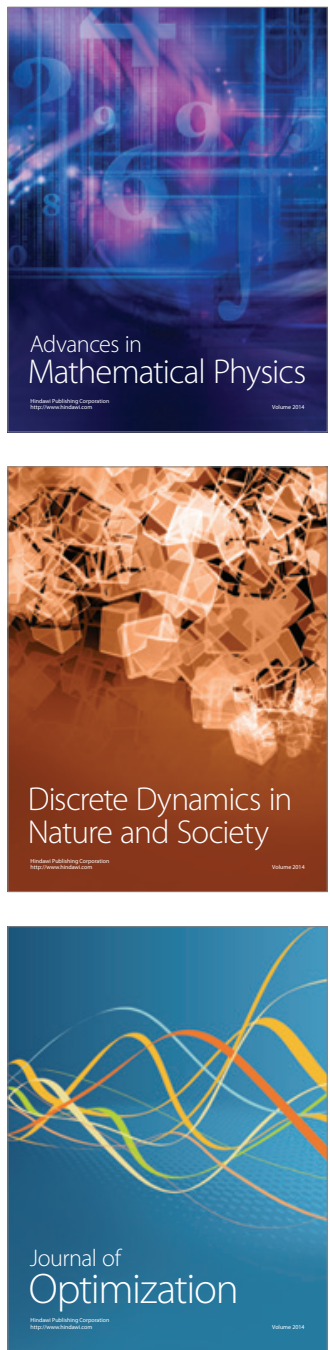